Sharif University of Technology
Scientia Iranica
Transactions E: Industrial Engineering
hCIENTIA

\title{
Extended economic production quantity models with preventive maintenance
}

\author{
H. Mokhtari ${ }^{\mathrm{a}, *}$ and J. Asadkhani ${ }^{\mathrm{b}}$ \\ a. Department of Industrial Engineering, Faculty of Engineering, University of Kashan, Kashan, P.O. Box 8731753153, Iran. \\ b. Department of Management and Entrepreneurship, Faculty of Humanities, University of Kashan, Kashan, P.O. Box 1471835191, \\ Iran.
}

Received 6 June 2018; received in revised form 24 February 2019; accepted 27 April 2019

\section{KEYWORDS}

Preventive

maintenance;

Production-inventory

system;

Defective process;

Manufacturing

planning;

Defective items.

\begin{abstract}
This paper generalizes the Economic Production Quantity (EPQ) model in the process manufacturing industry by incorporating regular Preventive Maintenance (PM) activities into classic EPQ model. The PM program improves the conditions of production to an acceptable level and avoids potential stops and disruptions. Therefore, it is a vital task in every production process. However, the EPQ model does not consider PM activities and thus, it is not applicable to real-world situations. A manufacturer that produced a product under the EPQ setting with a defective production process was considered in which every production cycle involved a number of sub-production cycles. Two models were developed based on the disposal time of defective items to determine the optimal number of subproduction cycles. In Model I, the disposal of defective items was performed once per cycle at the end of each production cycle, while in Model II, the disposal of defective items was performed multiple times per cycle at the end of each sub-production cycle. The total cost functions were derived for each model separately and then, simple solution algorithms were designed. A numerical example was dealt with to evaluate the proposed models. The results illustrated that Model II was more cost effective than Model I.
\end{abstract}

(C) 2020 Sharif University of Technology. All rights reserved.

\section{Introduction}

Nowadays, manufacturing companies seek an appropriate production process plan to optimize production and inventory costs in order to be more competitive in marketplace. Numerous models of inventory systems have been presented in the literature so far. Among them, Economic Order Quantity (EOQ) is the first and the basic one. In traditional EOQ, the demand is deterministic and constant over the planning horizon

\footnotetext{
*. Corresponding author. Tel./Fax: +983155902476 E-mail addresses: mokhtari_ie@kashanu.ac.ir (H. Mokhtari); javad.asadkhani@grad.kashanu.ac.ir (J. Asadkhani)
}

doi: $10.24200 /$ sci. 2019.51199 .2055 and the order is received instantaneously. The model aims to delineate the optimal order quantity for items to minimize the total costs including holding and ordering. Since the holding and ordering costs behave inversely in basic EOQ, the total cost function is convex and hence, an intermediate amount of order quantity becomes optimal. By relaxing some basic assumptions or adding new assumptions to the traditional EOQ model, many versions of the inventory model have been proposed so far. The Economic Production Quantity (EPQ) is one of the earlier extensions of EOQ. In the basic EOQ, it is assumed that the order quantity is received at a moment with an infinite rate, while in EPQ, orders are received at a finite rate over time. The EPQ model, also known as economic manufacturing quantity, aims to determine the optimal production quantity for a process manufacturing facility. The 
objective of the EPQ is to minimize the total inventory and production costs.

The standard EPQ model has been extended in various directions until now. For example, Jain and Rathore [1] studied the economic production quantity model for deteriorating items considering price and stock-dependent demand. Pan et al. [2] proposed an EPQ model integrated with the process control problem. Khedlekar [3] proposed an EPQ model with varying demand and production system disruption. Wee et al. [4] considered an EPQ model with a renewal reward procedure for imperfect items. Moreover, Dash et al. [5] designed an EPQ for deteriorating inventories with time value of money and price-dependent demand. An imperfect EPQ problem was suggested by KarimiNasab and Sabri-Laghaie [6] with reworkable and nonreworkable items and random defectives. Nasr et al. [7] utilized differential equations for an EPQ model with deteriorating raw materials. In addition, PachecoVelazquez and Cardenas-Barron [8] mentioned an EPQ problem by considering inventory costs of the raw materials and finished items, separately. Additionally, Jawad et al. [9] analyzed a sustainable EPQ using the laws of thermodynamics. In another work, a multiitem EPQ with fuzzy demand was proposed by Sadeghi et al. [10]. Moreover, Al-Salamah [11] suggested an EPQ model with quality control process where the items were subject to destructive or non-destructive inspection. Mokhtari et al. [12] proposed an EPQ model for perishable products with shortage and stockdependent demand. Mokhtari and Rezvan [13] studied an EPQ model in a multi-buyer and multi-product supply chain under partial backordering. Nasr et al. [14] proposed an EPQ model with maintenance and defective items where all defective items should be removed at the end of cycle. Moreover, Karmakar et al. [15] proposed a pollution-sensitive fuzzy EPQ model with a time-dependent rate of production. Nobil et al. [16] introduced a multi-machine multi-item EPQ model with several constraints including allocation of items, machine utilization, budget, production warehouse space, and capacity of each single machine. Wee et al. [17] examined the effect of two human factors on the economic quantity model. They concluded that both learning and fatigue played a key role in their proposed model.

Rezg et al. [18] discussed an inventory system in which Preventive Maintenance (PM) action would be activated upon a pre-planned scheme or a machine failure, whichever occurred first. Radhoui et al. [19] developed an inventory model with \%100 inspection policy. If the proportion of non-conforming items became more than a certain amount, the PM activities would be carried out. Otherwise, there should be no action. Liao and Sheu [20] developed an EPQ which conducted PM at the end of each production run period. In addition, PM might be either perfect or imperfect under known probability. Sana [21] investigated an inventory problem with imperfect production and PM actions. In each cycle, PM actions were executed when production process was completed. In the study mentioned above, the optimal production run time and optimal buffer inventory that could minimize the total cost per unit time were determined. Wee and Widyadana [22] proposed an EPQ model with deteriorating items the production process of which resulted in reworkable and scrap items. Moreover, PM was performed at the end of each rework process and the time was considered as a random parameter. Pal et al. [23] presented an EPQ model that applied PM to ensure that breakdown of the manufacture would not occur during the regular production process. Chen [24] incorporated an inventory/production problem into the PM activities. The regular production process produced imperfect items whose known fraction could be reworked. The PM was also imperfect and the number of imperfect items corresponded to the level of PM during the production cycle. Jafari and Makis [25] investigated an inventory problem whose production process deteriorated randomly. In this case, PM action would be activated upon a shift in production condition to a warning state. Sett et al. [26] studied an inventory problem with imperfect production and inspection errors. PM was carried out after production process and accordingly, shortage might occur. La Fata and Passannanti [27] incorporated a production-inventory system with PM policies in which breakdown might occur more than once at any age of the production unit. In addition, they assumed that safety stock would prevent shortage during the PM action, while it was possible that the system could not satisfy demand during corrective repair. Lai et al. [28] suggested an EPQ model whose maintenance policy, depending on the occurrence time of machine failure, comprised corrective and PM.

As a useful extension of EPQ, PM activities can be incorporated into the standard EPQ model. In real-world manufacturing processes, it is a common and frequent case that manufacturing machines are interrupted for maintenance activities. The standard EPQ models assume that the machine works continuously and there is no need for maintenance or service. However, it is not a realistic assumption and consequently, the standard EPQ is not applicable to many manufacturing processes. Moreover, the zero-defective process is rarely the case in practical situations and there are usually a number of defective items in any production process. This is also ignored by the standard EPQ model. This study designs two EPQ models where PM operations should be carried out at fixed time intervals and the process produces a number of defective items. When PM operation commences, 
the production is interrupted until the end of PM and then, it continues. Every production cycle involves a number of sub-production cycles to be optimally determined. The time of defective item disposal is considered a major parameter in the problem. In Model I, it is assumed that all defective items are disposed of at the end of production cycle altogether, while in Model II, the defective items are disposed of at the end of every sub-production cycle.

Finally, the objective of the present study is to answer the following questions:

- What is the impact of considering the imperfect production process and PM actions on the standard EPQ model?

- When should the defective items be disposed of from the inventory system?

- How many sub-productions should be considered to minimize the total cost per unit time in the proposed models?

- How many products are produced to minimize the total cost per unit time in the proposed models?

The rest of this paper is organized as follows. Section 2 reviews the standard $E P Q$ model as the base of the proposed models. Then, Section 3 describes the assumptions and notation. Sections 4 and 5 discuss the proposed models and their solution procedure, respectively. Section 6 presents a numerical example and finally, Section 7 concludes the paper.

\section{Basic model}

In this section, a brief description of the standard EPQ model as the base model of our study is presented. Then, the assumptions and notation employed to construct the models are presented. Finally, the two proposed models are described and distinguished.

\subsection{The standard EPQ model}

The EPQ model, regarded as an extension of the basic EOQ model, determines the optimal production quantity per cycle to minimize the total cost by balancing the production setup cost and inventory holding cost. The difference between these two models is that the EPQ assumes that the manufacturer produces its own quantity; therefore, the orders are available in an incremental manner at a finite rate, while the EOQ model assumes that the order quantity is received immediately after ordering. In the basic EPQ, demand for products is assumed continuous at a constant rate. The production process is made at regular intervals. The production setup cost is fixed and independent of the quantity produced. No discount is available. The replenishment is incrementally made via finite rate. Figure 1 shows the inventory level corresponding to

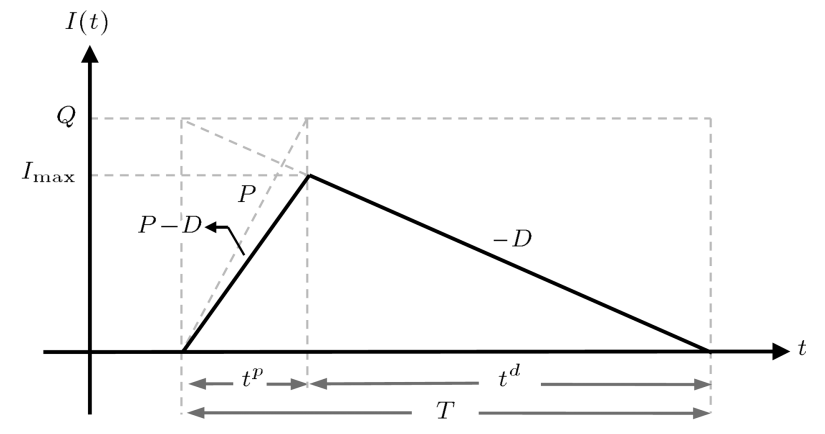

Figure 1. Inventory level under Economic Production Quantity (EPQ) model.

a single product under EPQ framework. At every inventory cycle $T$, the production is processed until the inventory reaches maximum level $I_{\max }$ in the production cycle $t^{p}$ and then, the stored inventory is consumed with the demand rate $D$ upon reaching zero in the depletion cycle $t^{d}$. The setup process of production incurs a fixed cost denoted by $A$ and the produced inventory can be stored with a holding cost per unit time denoted by $h$. The objective here is to find the EPQ, so that the total cost of inventory system involving setup and holding costs is minimized.

By considering the characteristics of the basic EPQ model as shown in Figure 1, the parameters of the model are obtained as $T=Q / D, t^{p}=Q / P$, $t^{d}=Q / D-Q / P$, and $I_{\max }=Q(1-D / P)$. The holding cost per cycle is $H C=h Q^{2}(1-D / P) /(2 D)$ and the setup cost per order is $O C=A$. The holding and setup costs per unit time can be calculated by dividing $H C$ and $O C$ through inventory cycle $T$, as $H C U=H C / T=h Q(1-D / P) / 2$, and $O C=A D / Q$. Therefore, the total cost including both holding and setup costs is calculated as follows:

$$
T C U=\frac{h Q}{2}\left(1-\frac{D}{P}\right)+\frac{A D}{Q},
$$

thus, the optimal production policy and total cost can be derived by setting the derivative of $T C U$ to zero:

$$
\begin{aligned}
& Q_{E P Q}^{*}=\sqrt{\frac{2 A D}{h\left(1-\frac{D}{P}\right)},} \\
& T C U\left(Q_{E P Q}^{*}\right)=\sqrt{2 A D h\left(1-\frac{D}{P}\right)} .
\end{aligned}
$$

\subsection{Inventory system description}

In the base model, there is a manufacturer who produces a product under EPQ framework. The product faces the external demand $D$, which is assumed to be deterministic and constant over time horizon. The manufacturer produces the product via a finite production rate $P$ to meet the demand of customers. 
Table 1. Characteristics of two models.

\begin{tabular}{cccccc}
\hline Model & EOQ/EPQ & $\begin{array}{c}\text { Imperfect } \\
\text { production process }\end{array}$ & Shortage & PM actions & $\begin{array}{c}\text { Number of } \\
\text { disposals per cycle }\end{array}$ \\
\hline Model I & EPQ & $\checkmark$ & $\times$ & $\checkmark$ & Single \\
Model II & EPQ & $\checkmark$ & $\times$ & $\checkmark$ & Multiple \\
\hline
\end{tabular}

The model comprises an infinite time horizon and zero lead time. In addition, any shortage is not allowed and purchase cost is fixed (with no discount option). In contrast to the standard EPQ model, PM activities should be carried out during fixed periods. During PM activities, the production is interrupted and then, it commences again at the end of the maintenance process. In general, an inventory cycle is composed of production and depletion cycles. The production cycle includes $m$, which is an integer greater than or equal to one, and consecutive sub-production cycles of fixed length $t_{S P}$, each followed by a PM activity of the fixed length $t_{P M}$. The depletion cycle is similar to that of standard EPQ model in which the stored inventory is depleted until it reaches zero. The inventory cost consists of holding and setup costs. The holding cost is per item per unit time, denoted by $h$, while the setup cost involves two terms. While every production cycle incurs a fixed setup cost $A_{1}$ (first term), each sub-production cycle incurs a fixed setup cost $A_{2}$ (second term). The number of defective items during every sub-production cycle is denoted by $S$. A list of assumptions and the notation used throughout the paper is presented in the following.

\subsubsection{Assumptions}

- Demand is deterministic and constant;

- Production rate is finite;

- Production rate is greater than the demand rate;

- Lead time is assumed to be zero;

- Shortage is not allowed;

- There is no discount option;

- Setup cost is fixed and incurs per production cycle and per sub-production cycle;

- Holding cost is per item per unit time;

- Production process is interrupted during PM activity;

- Duration of PM activities is fixed;

- Sub-production cycle time is fixed;

- Number of defective items per sub-production cycle is fixed.

\subsubsection{Notation}

$D \quad$ Demand rate of product

$P \quad$ Production rate of product
$A_{1} \quad$ Fixed setup cost per production cycle

$A_{2} \quad$ Fixed setup cost per sub-production cycle

$h \quad$ Holding cost of product per item per unit time

$t_{p} \quad$ Duration of production cycle

$t_{d} \quad$ Duration of depletion cycle

$T \quad$ Inventory cycle of product $\left(T=t_{p}+t_{d}\right)$

$t_{S P} \quad$ Duration of sub-production cycle

$t_{P M} \quad$ Duration of PM activity

$S \quad$ Number of defective items per sub-production cycle

$q \quad$ Number of sub-productions per cycle (decision variable)

$i \quad$ Index of sub-production cycle $(i=1,2, \cdots, q)$

$Q \quad$ Production quantity per cycle (dependent variable to $m$ )

In this study, two different models of EPQ with PM activities in terms of time are presented in which defective items are disposed of. In Model I, it is assumed that all defective items are disposed of at the end of a production cycle altogether, while in Model II, defective items are disposed of at the end of every subproduction cycle separately. In Model I, the removal of defective items occurs once per cycle, while in Model II, the removal of defective items occurs once per subproduction cycle, that is, multiple times per cycle. The details of both models are discussed in the subsequent subsections. Table 1 compares the main characteristics of both models.

\section{Model I: Disposal of defective items once per cycle}

In this section, the EPQ model is studied under PM activities in which defective items are removed from the system once at the end of the production cycle. Figure 2 depicts the behavior of the inventory system for this model.

To ensure feasibility and prevent shortage, we consider $(P-D) t_{S P}-\left(S+D t_{P M}\right)>0$. The total cost involves setup and holding costs. The setup cost $S C_{I}$ includes two terms per production cycle and per 


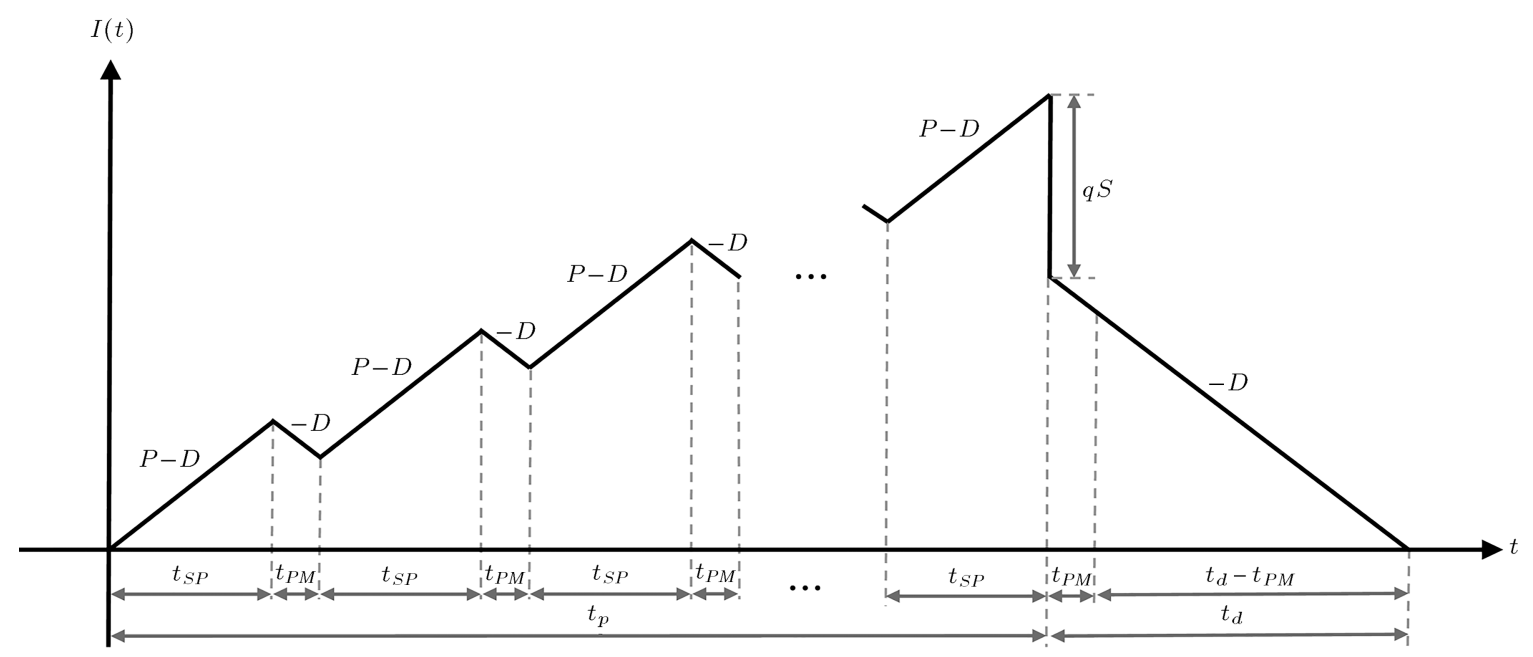

Figure 2. Inventory level for Model I.

sub-production cycle, which are:

$$
S C_{I}=A_{1}+q A_{2}
$$

To formulate the holding cost, first, the inventory level at the start of PM activity (end of current production process) at the $i$ th sub-production cycle is defined as $I_{i 1}$ and the inventory level at the end of PM activity (beginning of the next production process) at the $i$ th sub-production cycle as $I_{i 2}$ :

$$
\begin{aligned}
& I_{i 1}=i(P-D) t_{S P}-(i-1) D t_{P M}, \\
& \forall i=1,2, \cdots, q, \\
& I_{i 2}=i(P-D) t_{S P}-i D t_{P M}, \\
& \quad \forall i=1,2, \cdots, q .
\end{aligned}
$$

By using $I_{i 1}$ and $I_{i 2}$, the area under inventory level in Figure $2\left(A_{I}\right)$, as total inventory held in Model I, is calculated as follows:

$$
\begin{aligned}
A_{I}= & \sum_{i=1}^{q}\left[\left(\frac{I_{i 1}+I_{(i-1) 2}}{2}\right) t_{S P}+\left(\frac{I_{i 1}+I_{i 2}}{2}\right) t_{P M}\right] \\
& -q S t_{P M}+\frac{\left(I_{q 2}-q S\right)\left(t_{d}-t_{P M}\right)}{2}
\end{aligned}
$$

where $I_{02}=0$. Then, the holding cost can be calculated as $H C_{I}=h A_{I}$. Before calculating the holding cost, note that $t_{d}-t_{P M}$ can be written in terms of $I_{q 2}$ as shown in the following:

$$
t_{d}-t_{P M}=\frac{I_{q 2}-q S}{D} .
$$

Therefore, by substituting the $I_{i 1}, I_{i 2}$, and $t_{d}-t_{P M}$ into $A_{I}$ and simplifying the result, the holding cost $H C_{I}$ is written as:

$$
\begin{aligned}
H C_{I}= & h\left\{\frac{t_{S P}}{2}\left(q^{2} t_{S P}(P-D)-D q t_{P M}(q-1)\right)\right. \\
& +\frac{t_{P M}}{2}\left(-D q^{2} t_{P M}+q t_{S P}(P-D)(q+1)\right) \\
& \left.-q S t_{P M}+\frac{\left(S q+q t_{S P}(D-P)+D q t_{P M}\right)^{2}}{2 D}\right\},
\end{aligned}
$$

therefore, the total cost $T C_{I}=S C_{I}+H C_{I}$ is summarized as given below:

$$
\begin{aligned}
T C_{I}= & A_{1}+q A_{2} \\
& +h\left\{\frac{t_{S P}}{2}\left(q^{2} t_{S P}(P-D)-D q t_{P M}(q-1)\right)\right. \\
& +\frac{t_{P M}}{2}\left(-D q^{2} t_{P M}+q t_{S P}(P-D)(q+1)\right) \\
& \left.-q S t_{P M}+\frac{\left(S q+q t_{S P}(D-P)+D q t_{P M}\right)^{2}}{2 D}\right\} .
\end{aligned}
$$

Finally, the total cost per unit time in Model I, $T C U_{I}$, is obtained by dividing $T C_{I}$ by the inventory cycle time $C T_{I}$. First, the cycle time is calculated through $C T_{I}=$ $q\left(t_{S P}+t_{P M}\right)+t_{d}-t_{P M}$, which is simplified as:

$$
C T_{I}=q\left(P t_{S P}-S\right) / D
$$

Then, the total cost per unit time of Model I is obtained as shown below.

$$
\begin{aligned}
T C U_{I}= & \frac{T C_{I}}{q\left(P t_{S P}-S\right) / D}=\frac{A_{1} D}{q\left(P t_{S P}-S\right)} \\
& +\frac{A_{2} D}{\left(P t_{S P}-S\right)}+\frac{h D}{\left(P t_{S P}-S\right)}
\end{aligned}
$$




$$
\begin{aligned}
& \times\left\{\frac{t_{S P}}{2}\left(q t_{S P}(P-D)-D t_{P M}(q-1)\right)\right. \\
& +\frac{t_{P M}}{2}\left(-D q t_{P M}+t_{S P}(P-D)(q+1)\right) \\
& \left.-S t_{P M}+\frac{q\left(S+t_{S P}(D-P)+D t_{P M}\right)^{2}}{2 D}\right\} .
\end{aligned}
$$

As observed, the total cost $T C U_{I}$ is a function of decision variable $q$ (the number of sub-production cycles per cycle). To achieve the optimal value of this variable, the derivative of total cost $T C U_{I}$ is set to zero:

$$
\begin{aligned}
\frac{\partial T C U_{I}}{\partial q}= & -\frac{A_{1} D}{q^{2}\left(P t_{S P}-S\right)} \\
& +\frac{h D}{\left(P t_{S P}-S\right)}\left\{\frac{t_{S P}}{2}\left(t_{S P}(P-D)-D t_{P M}\right)\right) \\
& +\frac{t_{P M}}{2}\left(-D t_{P M}+t_{S P}(P-D)\right) \\
& \left.+\frac{\left(S+t_{S P}(D-P)+D t_{P M}\right)^{2}}{2 D}\right\}=0,(13)
\end{aligned}
$$

by simplifying the above equation, we obtain:

$$
\begin{aligned}
\frac{A_{1}}{q^{2}}= & h\left\{\frac{t_{S P}}{2}\left(t_{S P}(P-D)-\left(D t_{P M}\right)\right)\right. \\
& +\frac{t_{P M}}{2}\left(-D t_{P M}+t_{S P}(P-D)\right) \\
& \left.+\frac{\left(S+t_{S P}(D-P)+D t_{P M}\right)^{2}}{2 D}\right\},
\end{aligned}
$$

which yields:

$$
q_{I}=\sqrt{\frac{2 A_{1} D}{h\left\{D F\left(t_{S P}+t_{P M}\right)+(F-S)^{2}\right\}}},
$$

where:

$$
F=(P-D) t_{S P}-D t_{P M}
$$

In addition, the number of sub-production cycles in Model I, i.e., $q_{I}$, is used to obtain the production quantity at each cycle of Model I as $Q_{I}=t_{S P} P q_{I}$.

For further analysis of the obtained solution, $q_{I}^{*}$ with respect to the standard EPQ formula is thoroughly investigated. In the standard EPQ, no PM activity is observed, and thus, PM duration is zero $\left(t_{P M}=0\right)$ and there are no defective items $(S=0)$. In this regard, $F=(P-D) t_{S P}$ and the production quantity $Q_{I}=t_{S P} P q_{I}$ is simplified to:

$$
Q_{I}=\sqrt{\frac{2 A_{1} D}{h\left(1-\frac{D}{P}\right)}},
$$

which is equal to the optimal production quantity in the basic $\operatorname{EPQ}\left(Q_{E P Q}^{*}\right)$. This completes the comparative analysis between the proposed Model I and standard EPQ.

Since the sub-production cycle is an integer $(q \geq$ 1), a simple algorithm is designed to find the feasible solution. Prior to presenting the algorithm, it is necessary to evaluate the convexity of the total cost function $T C U_{I}$. To this end, the second derivative of $T C U_{I}$ with respect to $q$ is calculated as follows:

$$
\frac{\partial^{2} T C U_{I}}{\partial q^{2}}=\frac{2 A_{1} D}{q^{3}\left(P t_{S P}-S\right)} .
$$

Given that $P t_{S P}-S>0$ in Model I, it can be concluded that $\frac{\partial^{2} T C U_{I}}{\partial q^{2}}>0$ and hence, the convexity is proved. Therefore, the following algorithm finds the feasible solution:

Step 1: Find $q_{I}$ using Eq. (15);

Step 2: If $q_{I}$ is less than 1 , set $q_{I}^{*}=1$ and stop the algorithm;

Step 3: If $q_{I}$ is not an integer, set $q_{1}=\left[q_{I}\right]$ and $q_{2}=$ $\left[q_{I}\right]+1$, where [.] calculates the integer part of $q_{I}$;

Step 4: Calculate the total costs associated with $q_{1}$ and $q_{2}$ as $T C U_{1}$ and $T C U_{2}$;

Step 5: Select the minimum total cost as $T C U=$ $\min \left\{T C U_{1}, T C U_{2}\right\}$ and set the corresponding $q\left(q_{1}\right.$ or $\left.q_{2}\right)$ as $q_{I}^{*}$.

Having determined the optimal number of subproductions per cycle $q_{I}^{*}$, we can find the optimal duration of production cycle $t_{p}^{*}$ and depletion cycle $t_{d}^{*}$ in Model I as $t_{p}^{*}=q_{I}^{*} t_{S P}+\left(q_{I}^{*}-1\right) t_{P M}$ and $t_{d}^{*}=t_{P M}+$ $\left\{q_{I}^{*}(P-D) t_{S P}-q_{I}^{*} D t_{P M}-q_{I}^{*} S\right\} / D$, respectively.

\section{Model II: Removal of defective items once per sub-production cycle}

This section discusses the case in which defective items are removed from the system at the end of every subproduction cycle. Figure 3 depicts the behavior of the inventory system for this model in general.

Similar to Model I, $(P-D) t_{S P}-\left(S+D t_{P M}\right)>0$ is regarded as the feasibility condition. In addition, the setup cost $S C_{I I}$ includes two terms per production cycle and per sub-production cycle, including:

$$
S C_{I I}=A_{1}+q A_{2} .
$$

In order to formulate the holding cost, first, the inventory level at the end of sub-production cycle (before 


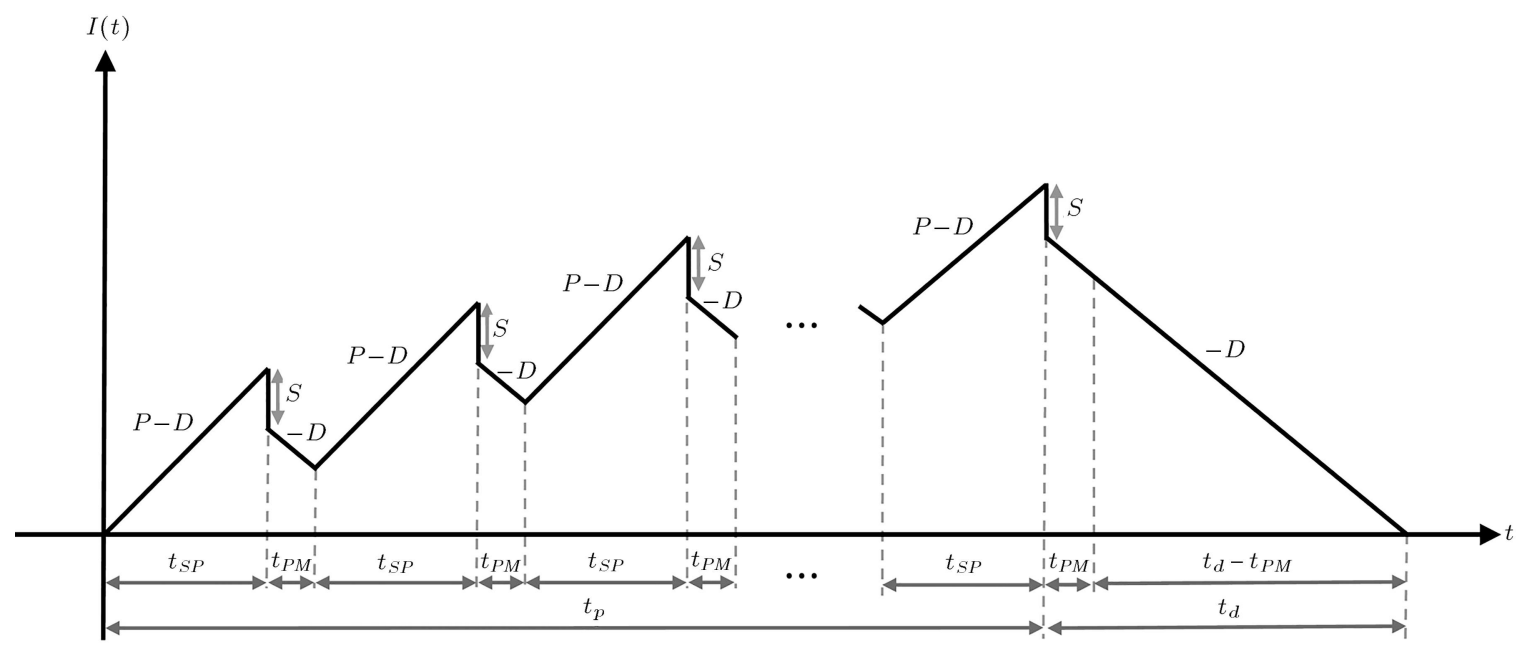

Figure 3. Inventory level for Model II.

starting maintenance) at the $i$ th sub-production cycle is defined as $I_{i 1}$, the inventory level at the start of PM activity at the $i$ th sub-production cycle as $I_{i 2}$, and the inventory level at the end of PM activity (start of next production process) at the $i$ th sub-production cycle as $I_{i 3}$ :

$$
\begin{aligned}
& I_{i 1}=i(P-D) t_{S P}-(i-1) S-(i-1) D t_{P M}, \\
& \forall i=1,2, \cdots, q, \\
& I_{i 2}=i(P-D) t_{S P}-i S-(i-1) D t_{P M}, \\
& \quad \forall i=1,2, \cdots, q, \\
& I_{i 3}=i(P-D) t_{S P}-i S-i D t_{P M}, \\
& \quad \forall i=1,2, \cdots, q .
\end{aligned}
$$

Using $I_{i 1}, I_{i 2}$, and $I_{i 3}$, the area under inventory level in Figure $3\left(A_{I I}\right)$, regarded as the total inventory hold in Model I, is calculated as follows:

$$
\begin{aligned}
A_{I I}= & \sum_{i=1}^{q}\left[\left(\frac{I_{i 1}+I_{(i-1) 3}}{2}\right) t_{S P}+\left(\frac{I_{i 2}+I_{i 3}}{2}\right) t_{P M}\right] \\
& -q S t_{P M}+\frac{I_{q 3}\left(t_{d}-t_{P M}\right)}{2}
\end{aligned}
$$

where $I_{03}=0$. Then, the holding cost can be calculated as $H C_{I I}=h A_{I I}$. To calculate $H C_{I I}$, note that $t_{d}-t_{P M}$ can be written in terms of $I_{q 3}$ :

$$
t_{d}-t_{P M}=\frac{I_{q 3}}{D}
$$

Therefore, by substituting $I_{i 1}, I_{i 2}, I_{i 3}$, and $t_{d}-t_{P M}$ into $A_{I I}$ and simplifying the result, the holding cost $H C_{I I}$ is obtained as:

$$
\begin{aligned}
H C_{I I}= & h\left\{-S\left(\frac{q t_{P M}(q+1)}{2}+\frac{q t_{S P}(q-1)}{2}\right)\right. \\
& -D t_{P M}\left(\frac{q^{2} t_{P M}}{2}+\frac{q t_{S P}(q-1)}{2}\right) \\
& +(P-D) t_{S P}\left(\frac{q^{2} t_{S P}}{2}+\frac{q t_{P M}(q+1)}{2}\right) \\
& \left.+\frac{q^{2}\left(S+D t_{P M}+t_{S P}(D-P)\right)^{2}}{2 D}\right\},
\end{aligned}
$$

therefore, the total cost $T C_{I I}=S C_{I I}+H C_{I I}$ is summarized as:

$$
\begin{aligned}
T C_{I I}= & A_{1}+q A_{2}+h\left\{-S\left(\frac{q t_{P M}(q+1)}{2}+\frac{q t_{S P}(q-1)}{2}\right)\right. \\
& -D t_{P M}\left(\frac{q^{2} t_{P M}}{2}+\frac{q t_{S P}(q-1)}{2}\right) \\
& +(P-D) t_{S P}\left(\frac{q^{2} t_{S P}}{2}+\frac{q t_{P M}(q+1)}{2}\right) \\
& \left.+\frac{q^{2}\left(S+D t_{P M}+t_{S P}(D-P)\right)^{2}}{2 D}\right\}
\end{aligned}
$$

Finally, the total cost per unit time in Model II, $T C U_{I I}$, is obtained through dividing $T C_{I I}$ by the inventory cycle time $C T_{I I}$. To this end, the cycle time is calculated through $C T_{I I}=q\left(t_{S P}+t_{P M}\right)+t_{d}-t_{P M}$, which is simplified to:

$$
C T_{I I}=q\left(P t_{S P}-S\right) / D,
$$

which is equal to $C T_{I}$. Then, the total cost per unit time of Model II is obtained as: 


$$
\begin{aligned}
T C U_{I I}= & \frac{T C}{q\left(P t_{S P}-S\right) / D}=\frac{A_{1} D}{q\left(P t_{S P}-S\right)} \\
& +\frac{A_{2} D}{\left(P t_{S P}-S\right)}+\frac{h D}{\left(P t_{S P}-S\right)} \\
& \times\left\{-S\left(\frac{t_{P M}(q+1)}{2}+\frac{t_{S P}(q-1)}{2}\right)\right. \\
& -D t_{P M}\left(\frac{q t_{P M}}{2}+\frac{t_{S P}(q-1)}{2}\right) \\
& +(P-D) t_{S P}\left(\frac{q t_{S P}}{2}+\frac{t_{P M}(q+1)}{2}\right) \\
& \left.+\frac{q\left(S+D t_{P M}+t_{S P}(D-P)\right)^{2}}{2 D}\right\} .
\end{aligned}
$$

To attain the optimal value for $q$, the derivative of total cost $T C U_{I I}$ is set to zero.

$$
\begin{aligned}
\frac{\partial T C U_{I I}}{\partial q}= & -\frac{A_{1} D}{q^{2}\left(P t_{S P}-S\right)} \\
& +\frac{h D}{\left(P t_{S P}-S\right)}\left\{-\frac{S}{2}\left(t_{P M}+t_{S P}\right)\right. \\
& -\frac{D t_{P M}}{2}\left(t_{P M}+t_{S P}\right) \\
& +(P-D) \frac{t_{S P}}{2}\left(t_{S P}+t_{P M}\right) \\
& \left.+\frac{1}{2 D}\left(S+D t_{P M}+t_{S P}(D-P)\right)^{2}\right\}=0 .
\end{aligned}
$$

By simplifying the above equation, the following equation is obtained:

$$
q_{I I}=\sqrt{\frac{2 A_{1} D}{h\left\{D G\left(t_{S P}+t_{P M}\right)+G^{2}\right\}}},
$$

where:

$$
G=(P-D) t_{S P}-\left(S+D t_{P M}\right) .
$$

Moreover, the production quantity at each cycle of Model II is obtained as $Q_{I I}=t_{S P} P q_{I I}$.

For further analysis of the obtained solution, $q_{I I}$ for the case of standard EPQ formula is investigated. In standard EPQ, we have $t_{P M}=0$ and $S=0$. Thus, $G=$ $t_{S P}(P-D)$ is achieved and the production quantity $Q_{I I}=t_{S P} P q_{I I}$ is simplified to:

$$
Q_{I I}=\sqrt{\frac{2 A_{1} D}{h\left(1-\frac{D}{P}\right)}}
$$

which is equal to the optimal production quantity in basic $\operatorname{EPQ}\left(Q_{E P Q}^{*}\right)$.

Since the sub-production cycle is an integer $(q \geq$ 1), a simple algorithm is designed to find the feasible solution. Similar to Mode I, it is required to evaluate the convexity of total cost function $T C U_{I I}$. To this end, the second derivative of $T C U_{I I}$ with respect to $q$ is calculated as follows:

$$
\frac{\partial^{2} T C U_{I}}{\partial q^{2}}=\frac{2 A_{1} D}{q^{3}\left(P t_{S P}-S\right)} .
$$

Since $P t_{S P}-S>0$, it can be concluded that $\frac{\partial^{2} T C U_{I I}}{\partial q^{2}}>$ 0 and hence, the convexity is proved. Furthermore, similar to Model I, by using the algorithm, the feasible solution for Model II is achieved as follows:

Step 1: Find $q_{I I}$ using Eq. (30);

Step 2: If $q_{I I}$ is less than 1 , set $q_{I I}^{*}=1$ and stop the algorithm;

Step 3: If $q_{I I}$ is not an integer, set $q_{1}=\left[q_{I I}\right]$ and $q_{2}=\left[q_{I I}\right]+1$ where [.] calculates the integer part of $q_{I I}$;

Step 4: Calculate the total costs associated with $q_{1}$ and $q_{2}$ as $T C U_{1}$ and $T C U_{2}$;

Step 5: Select the minimum total cost as $T C U=$ $\min \left\{T C U_{1}, T C U_{2}\right\}$ and set its corresponding $q\left(q_{1}\right.$ or $\left.q_{2}\right)$ as $q_{I I}^{*}$.

In addition, the optimal durations of production cycle $t_{p}^{*}$ and depletion cycle $t_{d}^{*}$ in Model II can be calculated as $t_{p}^{*}=q_{I I}^{*} t_{S P}+\left(q_{I I}^{*}-1\right) t_{P M}$ and $t_{d}^{*}=t_{P M}+\left\{q_{I I}^{*}(P-\right.$ $\left.D) t_{S P}-q_{I I}^{*} S-q_{I I}^{*} D t_{P M}\right\} / D$, respectively.

\section{Example and discussion}

This section discusses a numerical example in order to investigate the performance of the proposed EPQ models. Consider a manufacturer that produces a product under EPQ setting. The demand is deterministic and constant, $D=60$, and the production rate is finite $P=100$. In addition, the lead time is zero and shortage is not allowed. The setup costs are fixed and incurred per production cycle $A_{1}=240$ and per sub-production cycle $A_{2}=60$. The holding cost is calculated per item per unit time $h=2$. The duration of PM activities is fixed $t_{P M}=0.2$. The sub-production cycle time is fixed $t_{S P}=1.2$. The number of defective items per sub-production cycle is fixed $S=10$. Prior to solving this example, the total cost per unit times $T C U_{I}$ and $T C U_{I I}$ is derived as follows:

$$
\begin{aligned}
& T C U_{I}=\frac{370 q^{2}+480 q+1440}{11 q}, \\
& T C U_{I I}=\frac{286 q^{2}+564 q+1440}{11 q} .
\end{aligned}
$$




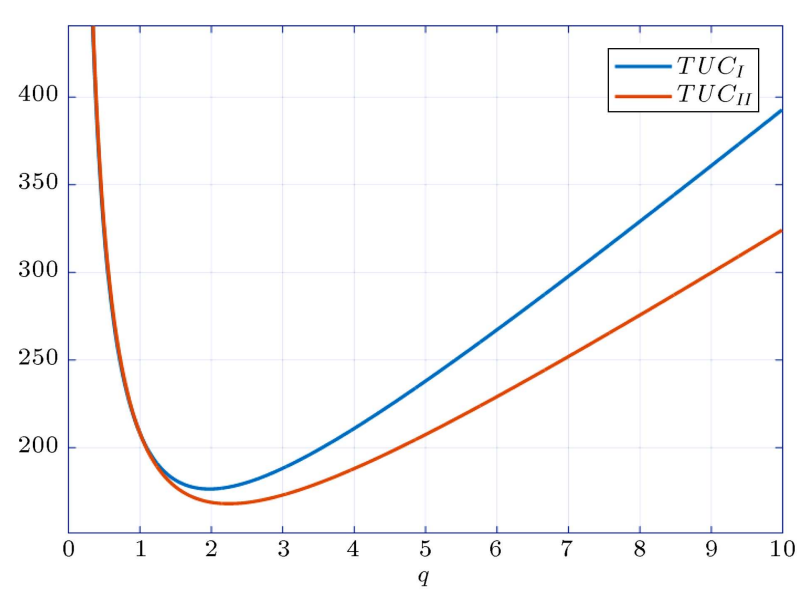

Figure 4. Total cost functions, $T C U_{I}$ and $T C U_{I I}$.

Figure 4 presents both total cost functions for further analysis of these two models.

Obviously, both cost functions are convex. Therefore, the minimum cost value of $q$ can be obtained by setting derivatives to zero.

$$
\begin{aligned}
& \frac{\partial T C U_{I}}{\partial q}=\frac{10\left(37 q^{2}-144\right)}{11 q^{2}}=0 \Rightarrow q_{I}=1.9728, \\
& \frac{\partial T C U_{I I}}{\partial q}=\frac{2\left(143 q^{2}-720\right)}{11 q^{2}}=0 \Rightarrow q_{I I}=2.2439 .
\end{aligned}
$$

In addition, these solutions can be obtained using Eqs. (15) and (30):

$$
\begin{aligned}
& F=((100-60) 1.2-60 \times 0.2)=36, \\
& q_{I}=\sqrt{\frac{2 \times 240 \times 60}{2\left\{60 \times 36 \times(1.2+0.2)+(36-10)^{2}\right\}}}=1.9728, \\
& G=((100-60) 1.2-(10+60 \times 0.2))=26 \\
& q_{I I}=\sqrt{\frac{2 \times 240 \times 60}{2\left\{60 \times 26 \times(1.2+0.2)+26^{2}\right\}}}=2.2439 .
\end{aligned}
$$

Since the number of sub-production cycles should be an integer greater than or equal to one, the obtained solutions are not efficient. To solve this example, the solution algorithms presented in the previous section are employed.

First, the algorithm for Model I is taken into account. To this end, the values of total cost $T C U_{I}$ for $q_{1}=[1.9728]=1$ and $q_{2}=[1.9728]+1=$ 2 are calculated as $T C U_{1}=208.18$ and $T C U_{2}=$ 176.36, respectively. Therefore, the minimum cost value is $T C U=\min \{208.18,176.36\}=176.36$, which is associated with $q_{2}=2$. In this respect, the optimal number of sub-production cycles in Model I is calculated as $q_{I}^{*}=2$. Then, the optimal production quantity per production cycle is calculated as $Q_{I}^{*}=$

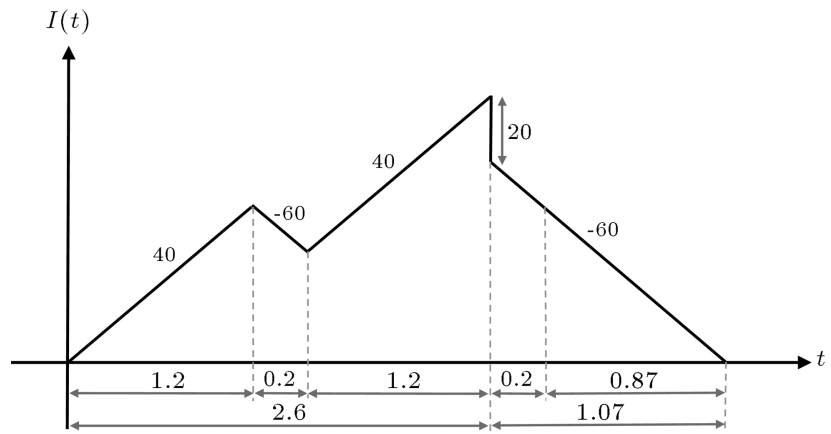

Figure 5. Optimal inventory level of the example (Model I).

$t_{S P} P q_{I}^{*}=240$. After determining the optimal number of sub-productions per cycle $q_{I}^{*}$, the optimal durations of production cycle $t_{p}^{*}$ and depletion cycle $t_{d}^{*}$ are calculated as $t_{p}^{*}=q_{I}^{*} t_{S P}+\left(q_{I}^{*}-1\right) t_{P M}=2.6$ and $t_{d}^{*}=t_{P M}+\left\{q_{I}^{*}(P-D) t_{S P}-q_{I}^{*} D t_{P M}-q_{I}^{*} S\right\} / D=$ 1.07 , respectively. Figure 5 shows the behavior of the inventory level for the optimal solution for Model I.

Figure 5 presents the obtained inventory levels in the first and second sub-production cycles. The inventory level at the beginning of $\mathrm{PM}$ activity at the first sub-production cycle is $I_{11}=(P-D) t_{S P}=48$, the inventory level at the end of PM activity at the first sub-production cycle is $I_{12}=(P-D) t_{S P}-D t_{P M}=36$, the inventory level at the start of PM activity at the second sub-production cycle is $I_{21}=2(P-D) t_{S P}-$ $D t_{P M}=84$, and the inventory level after disposal of defective items is $I_{21}-2 S=64$. Note that the maximum inventory level $I_{\max }$ is equal to $I_{21}$, i.e., $I_{\max }=84$.

Now, in order to find feasible solution for Model II, the values of total cost $T C U_{I I}$ for $q_{1}=$ [2.2439] $=2$ and $q_{2}=[2.2439]+1=3$ are calculated as $T C U_{1}=168.73$ and $T C U_{2}=172.91$, respectively. The minimum cost value is $T C U=\min \{168.73,172.91\}=$ 168.73 , which is associated with $q_{1}=2$. Therefore, the optimal number of sub-production cycles in Model II is $q_{I I}^{*}=2$ and the optimal production quantity per production cycle is calculated as $Q_{I I}^{*}=t_{S P} P q_{I I}^{*}=240$. Moreover, the optimal durations of production cycle $t_{p}^{*}$ and depletion cycle $t_{d}^{*}$ can be obtained using $t_{p}^{*}=$ $q_{I I}^{*} t_{S P}+\left(q_{I I}^{*}-1\right) t_{P M}=2.6$ and $t_{d}^{*}=t_{P M}+\left\{q_{I I}^{*}(P-\right.$ $\left.D) t_{S P}-q_{I I}^{*} S-q_{I I}^{*} D t_{P M}\right\} / D=1.07$, respectively. Figure 6 shows the behavior of inventory level for the optimal solution for Model II.

The inventory level at the end of production process in the first sub-production cycle is $I_{11}=$ $(P-D) t_{S P}=48$, the inventory level at the beginning of PM activity in the first sub-production cycle is $I_{12}=(P-D) t_{S P}-S=38$, the inventory level at the end of PM activity at the first sub-production cycle is $I_{13}=(P-D) t_{S P}-S-D t_{P M}=24$, the inventory level at the end of production process at the second sub- 


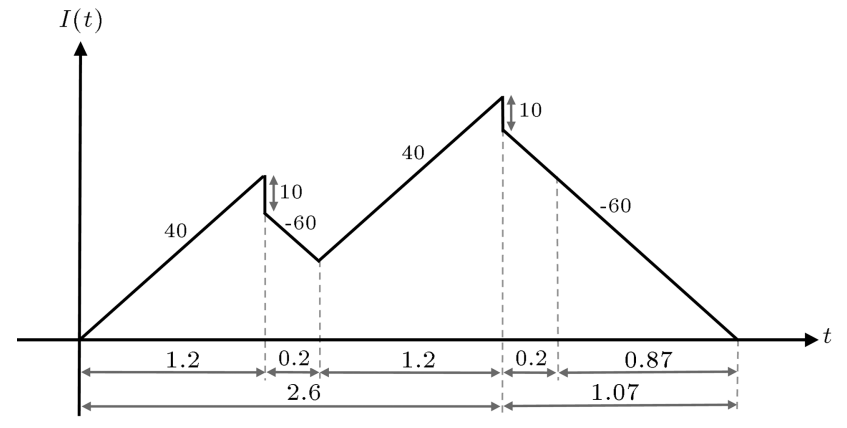

Figure 6. Optimal inventory level of the example (Model II).

production cycle is $I_{21}=2(P-D) t_{S P}-S-D t_{P M}=$ 74 , and the inventory level at the beginning of PM activity at the second sub-production cycle is $I_{22}=$ $2(P-D) t_{S P}-2 S-D t_{P M}=64$. Of note, maximum inventory level $I_{\max }$ is equal to $I_{21}$, i.e. $I_{\max }=74$.

A comparison of these two models indicates that Model II outperforms Model I in terms of total cost criterion due to a higher amount of inventory carried in the latter. In this model, the defective items remain in the system until the end of production cycle, while they are disposed of at the end of every sub-production cycle in Model II. However, these models show the same performance when the holding cost $h$ is small or the number of defective items is negligible. In addition, a practical recommendation for this example is that the managers can decide to plan multiple disposals of defective items at the appropriate time of each cycle. Note that the required time for defective items disposal corresponds to the number of sub-cycles. The numerical example results illustrate that optimal number of sub-cycles is calculated based on Model II. This decision can significantly decrease the total cost of the inventory system.

\section{Conclusions}

The Economic Production Quantity (EPQ) model, regarded as an extension of the basic Economic Order Quantity (EOQ) model, aims to determine the optimal production quantity to minimize the total cost. This paper extended the standard EPQ model by incorporating regular Preventive Maintenance (PM) activities. A manufacturer with a single product under EPQ setting and defective production process was taken into account. Every production cycle involved a number of sub-production cycles. According to the disposal time of defective items, two models were proposed to determine the optimal number of sub-production cycles. In the first model, the defective items were disposed of at the end of production cycle, while in the second model, defective items were disposed of at the end of sub-productions. The total cost functions were derived for each model separately. The convexity of cost functions was discussed and then, closed-form solutions for models were obtained. To guarantee feasibility, simple solution algorithms were designed. A numerical example was presented and implemented step by step in order to illustrate the procedure of the solution approaches. The results indicated that the second model guaranteed minimum total cost due to a higher amount of inventory held by the first model. In special cases, such as small holding cost or negligible number of defective items produced, both models showed the same performance in terms of total cost.

The incorporation of PM into classical inventory models is a new direction for future researchers. This paper can be extended in various directions. For instance, one can consider the production process with uncertain parameters such as the number of defective items produced. Moreover, it can be interesting to design a complicated problem in which PM is also a decision variable in terms of time of occurrence, PM duration, and selection among multi-level PMs. In addition, it can be assumed that PM action is imperfect and error-prone. In this case, probability of PM error occurrence follows the known probability density function. Imperfect PM may result in shifting the production system to an out-of-control state and cause an increase in the fraction of defective items in the production lot.

\section{References}

1. Jain, M. and Rathore, S. "Economic production quantity models with Shortage, price and stock-dependent Demand for deteriorating items", IJE Transactions, 20(2), pp. 159-166 (2007).

2. Pan, E., Jin, Y., Wang, S., and Cang, T. "An integrated EPQ model based on a control chart for an imperfect production process", International Journal of Production Research, 50(23), pp. 6999-7011 (2012).

3. Khedlekar, U.K. "A disruption production model with exponential demand", International Journal of Industrial Engineering Computations, 3(4), pp. 607-616 (2012).

4. Wee, H.M., Wang, W.T., and Yang, P.C. "A production quantity model for imperfect quality items with shortage and screening constraint", International Journal of Production Research, 51(6), pp. 1869-1884 (2013).

5. Dash, B., Pattnaik, M., and Pattnaik, H. "Deteriorated Economic Production Quantity (EPQ) model for declined quadratic demand with time value of money and shortages", Applied Mathematical Sciences, 8(73), pp. 3607-3618 (2014).

6. Karimi-Nasab, M. and Sabri-Laghaie, K. "Developing approximate algorithms for EPQ problem with process compressibility and random error in produc- 
tion/inspection", International Journal of Production Research, 52(8), pp. 2388-2421 (2014).

7. Nasr, W.W., Salameh, M.K., and Moussawi-Haidar, L. "Integrating the economic production model with deteriorating raw material over multi-production cycles", International Journal of Production Research, 52(8), pp. 2477-2489 (2014).

8. Pacheco-Velazquez, E.A. and Cárdenas-Barrón, L.E. "An economic production quantity inventory model with backorders considering the raw material costs", Scientia Iranica, 23(2), pp. 736-746 (2016).

9. Jawad, H., Jaber, M.Y., Bonney, M., and Rosen, M.A. "Deriving an exergetic economic production quantity model for better sustainability", Applied Mathematical Modelling, 40(11-12), pp. 6026-6039 (2016).

10. Sadeghi, J., Akhavan Niaki, S.T., Malekian, M.R., and Sadeghi, S. "Optimizing multi-item economic production quantity model with trapezoidal fuzzy demand and backordering: two tuned meta-heuristics", European Journal of Industrial Engineering, 10(2), pp. 170-195 (2016).

11. Al-Salamah, M. "Economic production quantity in batch manufacturing with imperfect quality, imperfect inspection, and destructive and non-destructive acceptance sampling in a two-tier market", Computers \& Industrial Engineering, 93, pp. 275-285 (2016).

12. Mokhtari, H., Naimi-Sadigh, A., and Salmasnia, A. "A computational approach to economic production quantity model for perishable products with backordering shortage and stock-dependent demand", Scientia Iranica, 24(4), pp. 2138-2151 (2017).

13. Mokhtari, H. and Rezvan, M.T. "A single-supplier, multi-buyer, multi-product VMI production-inventory system under partial backordering", Operational Research, 20(1), pp. 1-21 (2020). DOI: 10.1007/s12351017-0311-z

14. Nasr, W.W., Salameh, M., and Moussawi-Haidar, L. "Economic production quantity with maintenance interruptions under random and correlated yields", International Journal of Production Research, 55(16), pp. 4544-4556 (2017).

15. Karmakar, S., Kumar De, S., and Goswami, A. "A pollution sensitive dense fuzzy economic production quantity model with cycle time dependent production rate", Journal of Cleaner Production, 154, pp. 139150 (2017).

16. Nobil, A.H., Sedigh, A.H.A., and Cárdenas-Barrón, L.E. "Multi-machine economic production quantity for items with scrapped and rework with shortages and allocation decisions", Scientia Iranica, Transaction E, Industrial Engineering, 25(4), pp. 2331-2346 (2018).

17. Wee, H.M., Fu, K., Chen, Z., and Zhang, Y. "Optimal production inventory decision with learning and fatigue behavioral effect in labor intensive manufacturing", Scientia Iranica, 27(2), pp. 1-42 (2020). DOI: 10.24200/SCI.2018.50614.1788
18. Rezg, N., Dellagi, S., and Chelbi, A. "Joint optimal inventory control and preventive maintenance policy", International Journal of Production Research, 46(19), pp. 5349-5365 (2008).

19. Radhoui, M., Rezg, N., and Chelbi, A. "Integrated model of preventive maintenance, quality control and buffer sizing for unreliable and imperfect production systems", International Journal of Production Research, 47(2), pp. 389-402 (2009).

20. Liao, G.L. and Sheu, S.H. "Economic production quantity model for randomly failing production process with minimal repair and imperfect maintenance", International Journal of Production Economics, 130(1), pp. 118-124 (2011).

21. Sana, S.S. "Preventive maintenance and optimal buffer inventory for products sold with warranty in an imperfect production system", International Journal of Production Research, 50(23), pp. 6763-6774 (2012).

22. Wee, H.M. and Widyadana, G.A. "Economic production quantity models for deteriorating items with rework and stochastic preventive maintenance time", International Journal of Production Research, 50(11), pp. 2940-2952 (2012).

23. Pal, B., Sana, S.S., and Chaudhuri, K. "A mathematical model on EPQ for stochastic demand in an imperfect production system", Journal of Manufacturing Systems, 32(1), pp. 260-270 (2013).

24. Chen, Y.C. "An optimal production and inspection strategy with preventive maintenance error and rework", Journal of Manufacturing Systems, 32(1), pp. 99-106 (2013).

25. Jafari, L. and Makis, V. "Optimal lot-sizing and maintenance policy for a partially observable production system", Computers \& Industrial Engineering, 93, pp. 88-98 (2016).

26. Sett, B.K., Sarkar, S., and Sarkar, B. "Optimal buffer inventory and inspection errors in an imperfect production system with preventive maintenance", The International Journal of Advanced Manufacturing Technology, 90(1-4), pp. 545-560 (2017).

27. La Fata, C.M. and Passannanti, G. "A simulated annealing-based approach for the joint optimization of production/inventory and preventive maintenance policies", The International Journal of Advanced Manufacturing Technology, 91(9-12), pp. 3899-3909 (2017).

28. Lai, X., Chen, Z., and Bidanda, B. "Optimal decision of an economic production quantity model for imperfect manufacturing under hybrid maintenance policy with shortages and partial backlogging", International Journal of Production Research, 57(19), pp. 1-25 (2019). DOI: 10.1080/00207543.2018.1562249

\section{Biographies}

Hadi Mokhtari is currently an Associate Professor of Industrial Engineering at the University of Kashan, 
Iran. His current research interests include the applications of operations and artificial intelligence techniques to the areas of project scheduling, production scheduling, manufacturing supply chains, engineering economics, and inventory control problems. He has published several papers in international journals such as Computers and Operations Research, International Journal of Production Research, Applied Soft Computing, Neurocomputing, International Journal of Ad- vanced Manufacturing Technology, IEEE Transactions on Engineering Management, and Expert Systems with Applications.

Javad Asadkhani is currently an MSc student in Executive MBA at the University of Kashan, Iran. His current research interests include the applications of operations research to inventory control and supply chain problems. 